$13^{\text {th }}$ International Conference on

AEROSPACE SCIENCES \& AVIATION TECHNOLOGY,

ASAT- 13, May 26 - 28, 2009, E-Mail: asat@mtc.edu.eg

Military Technical College, Kobry Elkobbah, Cairo, Egypt

Tel : +(202) $24025292-24036138$, Fax: +(202) 22621908

\title{
A Measurement Weighting Method for Tracking Military Objects
}

\author{
Ashraf M. Aziz , M. Moghazy ${ }^{*}$, Ahmed M. ElBakly ${ }^{*}$, and M. H. AbdelAzeem*
}

\begin{abstract}
Prior methods for tracking multiple military objects include various optimal and suboptimal two-dimensional assignment algorithms which make nearest-neighbour measurement-to-track association. This method works reasonably well in case of small number of targets and high signal to noise ratio. Another method is to assign a weight for each measurement and use a weighted centroid of those measurements to update the track. This method of weighting the measurements is known as all-neighbour and overcomes the disadvantages of the nearest-neighbour data association. Unfortunately, the computational complexity of an optimal all-neighbour data association technique limits its practical realization using even the fastest computers available. For this reason, many different tracking techniques have been developed which sacrifice optimal performance for the sake of computational feasibility. This paper proposes a new computationally feasible measurement weighting method to the problem of multiple targets tracking in a noisy environment. Computer simulation results indicate that the proposed weighting method successfully tracks multiple targets with a lower computational complexity and a little prior knowledge.
\end{abstract}

Keywords: Nearest-neighbor association, All-neighbor association, Kalman Filter, Tracking, Measurement-to-tracks association.

\section{Introduction}

The main objective of any tracking system is to estimate and predict the target tracks. This is of interest in both civilian applications, such as civilian air traffic control, and military applications, such as air defense systems [1, 2]. When tracking multiple targets, data association decides which of the received measurements to use to update each target track. There are two solutions to the data association problem; the neighbor-neighbor filter (NNF) and the all-neighbor filter (ANF) [3]. In the NNF, one measurement, at most, can be used to update a given target track. The measurement that is closet to the predicted target measurement is used to update a given target track. In this approach, measurements are assigned to existing tracks in such a way to optimize an overall association measure. Blackman [4] provides an excellent description of NNF tracking techniques. The performance of the NNF does not work reasonably in case of low signal to noise ratio and large number of targets. Instead of using only one measurement among all the received ones and discarding the others, the ANF uses all of the validated measurements with different weights. The result is that the updated target estimate may contain contributions from more than one measurement with some association weights [3].

\footnotetext{
${ }^{*}$ Egyptian Armed Forces
} 
The multiple hypothesis tracker (MHT) is recognized as the theoretically optimum approach in Bayesian sense under idealized modeling assumptions. It based upon use of the a posteriori probability as calculated using Bayes' rule. Unfortunately, the computational complexity of the MHT limits its practical realization using even the fastest computers available [5]. Furthermore, a priori knowledge of the signal environment is required. For this reason, many different tracking techniques have been developed which sacrifice optimal performance for the sake of computational feasibility. Bar-shalom and Fortman [2] developed the joint probabilistic data association filter for tracking multiple targets. It is used to track multiple targets by evaluating the measurement-to-track probabilities for all measurements and all tracks for the last scan of measurements and combining the estimates based on the individual probability weights. Unfortunately, the implementation of this filter is infeasible due to the computational complexity required to perform tracking [6-8].

This paper proposes a new weighting method for tracking multiple targets. It incorporates all of the validated measurements, in a gate, with different fuzzy weights to update the state estimates. The proposed method is able to track multiple targets with a little prior knowledge and a lower computational complexity. The remainder of this paper is organized as follows. Problem formulation of multitarget tracking is addressed in Section 2. The proposed weighting method is presented in Section 3. To demonstrate the feasibility and efficiency of the proposed method, simulated multiple targets tracking, based on Monte Carlo simulations, is reported in Section 4. Performance comparison with NNF is also presented in Section 4. Finally, Section 5 contains conclusions.

\section{Problem Formulation}

Consider the case of multiple $\left(n_{t}\right)$ target tracks in the presence of nearby objects. Assume validation gates of the targets are defined in some way, and $n_{m}$ validated measurements are found inside them. A validation gate of a target is the gate in which the true measurement will appear with high probability. The target validation gates indicate the feasible joint events. Define the validation matrix as [9-12]:

$$
W=\left[w_{i j}\right], i=1,2, \ldots \ldots ., n_{t}, j=1,2, \ldots \ldots . ., n_{m},
$$

with binary elements $w_{i j}$. If measurement $j$ lies in the validation gate of target $i$, the corresponding element $w_{i j}=1$. Thus

$$
w_{i j}= \begin{cases}1, & \text { if measurement } j \text { falls within the validation gate of target } i \\ 0, & \text { otherwise }\end{cases}
$$

For an example, suppose that $n_{t}=2$ (two targets), and $n_{m}=4$ (four measurements fall within the validation gates of the two targets), as depicted in Fig.1. Figure 1 shows that measurement 1 falls within the gate of target $1\left(\omega_{11}=1\right)$, and outside the gate of target 2 $\left(\omega_{21}=0\right)$. The scenario of Fig.1 can be represented by the following validation matrix [1315]:

$$
W(i, j)=\left(\begin{array}{llll}
1 & 0 & 1 & 1 \\
0 & 1 & 1 & 0
\end{array}\right), \quad i=1,2, j=1,2,3,4 .
$$


Suppose that the number of validated measurements $\left(n_{m}\right)$, defined as $\left\{\mathbf{z}_{1}(k), \mathbf{z}_{2}(k), \ldots \ldots \ldots . . ., \mathbf{z}_{n_{m}}(k)\right\}$,

are received at scan $k$. The dynamical and measurement models of the $i^{\text {th }}$ target, $i=1,2, \ldots \ldots, n_{t}$, are defined as $[16-18]$

$\mathbf{x}_{i}(k+1)=\mathbf{F}_{i} \mathbf{x}_{i}(k)+\mathbf{v}_{i}(k)$,

$\mathbf{z}_{i}(k)=\mathbf{H}_{i}(k) \mathbf{x}_{i}(K)+\mathbf{w}_{i}(k)$,

where $\mathbf{x}_{i}(k)$ is an $n$ dimensional state vector of the $i^{\text {th }}$ target at scan $k, \mathbf{z}_{i}(k)$ is an $m$ dimensional attribute vector, $\mathbf{v}_{i}(k)$ is a noise input vector, $\mathbf{w}_{i}(k)$ is a measurement noise vector, $\mathbf{F}_{i}$ is an $n \times n$ state transition matrix, and $\mathbf{H}_{i}$ is an $m \times n$ measurement matrix. The process noise and the measurement noise are assumed to be uncorrelated, zero mean Gaussian with covariance matrices

$\mathbf{Q}_{i}(k)=\operatorname{Cov}\left(\mathbf{v}_{i}(k)\right)$,

$\mathbf{R}_{i}(k)=\operatorname{Cov}\left(\mathbf{w}_{i}(k)\right)$.

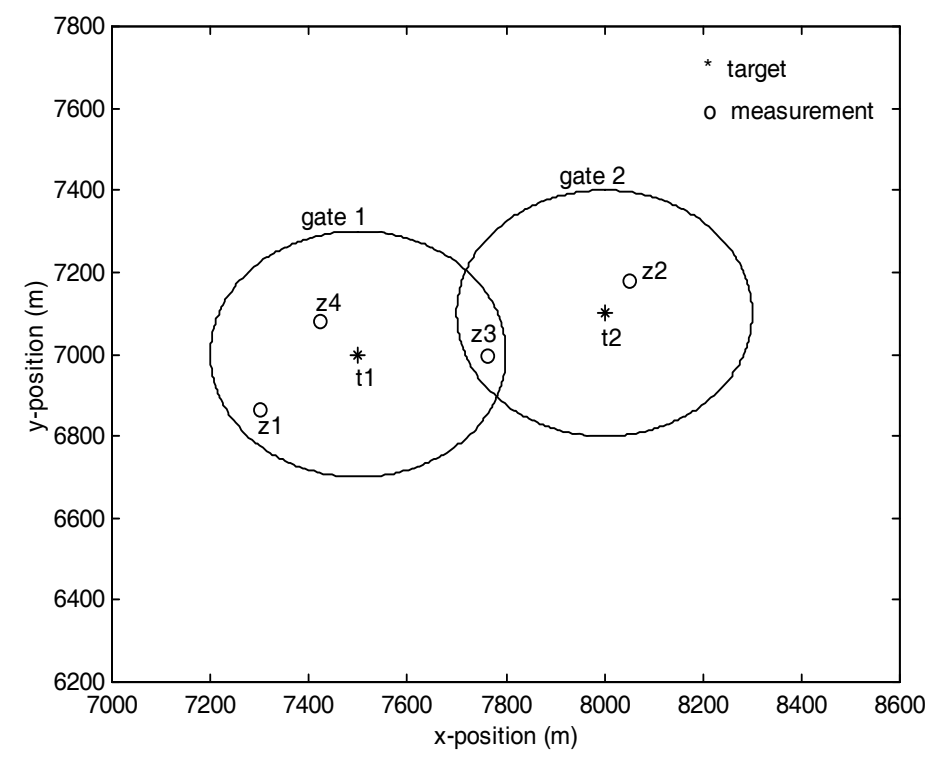

Fig. 1 Problem formulation with four measurements and two targets

Given $n_{m}$ measurements, it is required to determine the track estimates for all existing targets ( $n_{t}$ targets). It is difficult to determine precisely which target corresponds to each of the closely spaced received measurements. The problem is to construct the target state estimate by using the uncertain measurements originating from the targets in track. In a noise-free environment, each target track $i$ is predicted and updated based on correct measurements as follows $[1-4,16,18]$ (assuming the Kalman filter least squares estimator):

$\hat{\mathbf{x}}_{i}(k+1 \mid k)=\mathbf{F}_{i} \hat{\mathbf{x}}_{i}(k \mid k)$, 


$$
\begin{aligned}
& \mathbf{P}_{i}(k+1 \mid k)=\mathbf{F}_{i} \mathbf{P}_{i}(k) \mathbf{F}_{i}^{\prime}+\mathbf{Q}_{i}(k), \\
& \hat{\mathbf{x}}_{i}(k+1 \mid k+1)=\hat{\mathbf{x}}_{i}(k+1 \mid k)+\mathbf{K}_{i}(k+1) \tilde{\mathbf{z}}_{i}(k+1), \\
& \mathbf{P}_{i}(k+1 \mid k+1)=\left[\mathbf{I}-\mathbf{K}_{i}(k+1) \mathbf{H}_{i}(K+1)\right] \mathbf{P}_{i}(k+1 \mid k),
\end{aligned}
$$

where the Kalman filter gain $\mathbf{K}_{i}(k)$ and the innovation $\widetilde{\mathbf{z}}_{i}(k+1)$ are given by

$$
\begin{aligned}
& \mathbf{K}_{i}(k+1)=\mathbf{P}_{i}(k+1 \mid k) \mathbf{H}_{i}^{\prime}(k+1)\left[\mathbf{H}(k+1) \mathbf{P}_{i}(k+1 \mid k) \mathbf{H}_{i}^{\prime}(k+1)+\mathbf{R}_{i}(k+1)\right]^{-1}, \\
& \widetilde{\mathbf{z}}_{i}(k+1)=\mathbf{z}_{i}(k+1)-\mathbf{H}_{i}(k+1) \hat{\mathbf{x}}_{i}(k+1 \mid k) .
\end{aligned}
$$

The covariance matrix of the innovation is given by

$\mathbf{S}_{i}(k+1)=\mathbf{H}_{i}(k+1) \mathbf{P}_{i}(k+1 \mid k) \mathbf{H}_{i}(k+1)+\mathbf{R}_{i}$.

The problem is how to assign one measurement, from all, to update a target track $[13,18]$.

\section{Proposed Weighting Method}

Given a number of received measurements $n_{m}$, it is required to group them into $n_{t}$ targets according to some similarity measure. Define the error between a received measurement $j$ and a target $i$ as the square error (inner product induced norm):

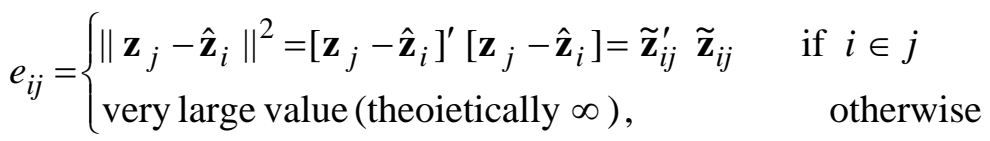

where $\tilde{\mathbf{z}}_{i j}$ is called the innovation vector between measurement $j$ and target $i$. Define the error matrix e as

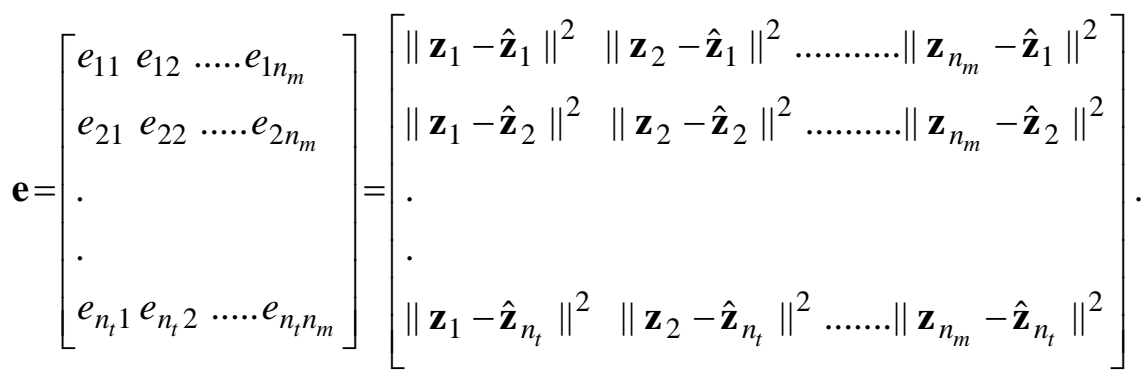

The similarity measures between all targets and all measurements $\left(s_{i j}\right)$ are determined such that the sum of the square errors $\left(E_{s}\right)$ is minimum, i.e. to minimize

$$
E_{s}=\sum_{j=1}^{n_{m}} \sum_{i=1}^{n_{t}}\left(s_{i j}\right)^{2} e_{i j}, i=1,2, \ldots \ldots ., n_{t}, j=1,2, \ldots \ldots \ldots, n_{v},
$$

where each error is weighted by the square of the corresponding degree of similarity measure. 
This problem can be treated as a clustering problem and can be solved using fuzzy clustering techniques. The solution for $\left\{s_{i j}, \forall i, j\right\}$ which achieves a minimum value of the square error is given by [7]:

$$
s_{i j}=\frac{\left(1 / e_{i j}\right)^{1 / 2}}{\sum_{\ell=1}^{n_{t}}\left(1 / e_{\ell j}\right)^{1 / 2}}, i=1,2, \ldots \ldots ., n_{t}, j=1, \ldots \ldots \ldots . ., n_{m},
$$

where $s_{i j}$ represents the similarity between target $i$ and measurement $j$. Note that if $e_{i j}=\infty$, the corresponding similarity measure $s_{i j}$ will be 0 . The similarity values are normalized such that, for a given track $i$, the contributions of all measurements equal unity, i.e.,

$$
\sum_{j=1}^{n_{m}} s_{i j}=1, i=1,2, \ldots \ldots . ., n_{t} .
$$

According to the normalized weights of the similarity measures $\left\{s_{i j}, \forall i, j\right\}$, the state estimate of target $i$ is updated using the equation

$$
\hat{\mathbf{x}}_{i}(k+1 \mid k+1)=\hat{\mathbf{x}}_{i}(k+1 \mid k)+\mathbf{K}_{i}(k+1) \sum_{j=1}^{n_{m}} s_{i j} \tilde{\mathbf{z}}_{i j}(k+1), \quad i=1,2, \ldots \ldots \ldots, n_{t} .
$$

The update covariance matrix is obtained as.

$$
\mathbf{P}_{i}(k+1 \mid k+1)=\mathbf{P}_{i 1}(k+1 \mid k+1)+\mathbf{P}_{i 2}(k+1 \mid k+1),
$$

where $\mathbf{P}_{i 1}(k+1 \mid k+1)$ and $\mathbf{P}_{i 2}(k+1 \mid k+1)$ are defined as

$$
\begin{aligned}
\mathbf{P}_{i 1}(k+1 \mid k+1) & =\left[\mathbf{I}-\mathbf{K}_{i}(k+1) \mathbf{H}_{i}(K+1)\right] \mathbf{P}_{i}(k+1 \mid k), \\
\mathbf{P}_{i 2}(k+1 \mid k+1) & =\mathbf{K}_{i}(k+1)\left[\sum_{j=1}^{n_{m}} s_{i j} \widetilde{\mathbf{z}}_{i j}(k+1) \tilde{\mathbf{z}}_{i j}^{\prime}-\tilde{\mathbf{z}}_{i}(k+1) \widetilde{\mathbf{z}}_{i}^{\prime}(k+1)\right] \mathbf{K}_{i}^{\prime}(k+1) .
\end{aligned}
$$

In summary, the proposed method consists of the following steps:

(1) Construct the validation matrix $W=\left[w_{i j}\right], i=1,2, \ldots, n_{t}, j=1,2, \ldots, n_{m}$, with binary elements $w_{i j}$ such that $w_{i j}=1$ if $j \in i$, and 0 otherwise.

(2) Based on the validation matrix $W$, evaluate the error matrix using (16) and construct the similarity weights $\left\{s_{i j}, \forall i, j\right\}$ using (18).

(3) Normalize the similarity weights $\left\{s_{i j}, \forall i, j\right\}$ to satisfy (19).

(4)Update the state estimate and covariance matrix according to (20)-(23). 


\section{Performance Evaluation Using Monte Carlo Simulations}

We consider two simulated examples. The first example considers the case of four crossing targets. We assume that the targets are moving in straight lines without acceleration. Measurement data are created by simulating the actual target motion in two dimensions and then adding noise to the true measurements. The targets motion model is defined by (4) and (5), where the state transition matrix $\mathbf{F}$ is given by

$$
\mathbf{F}=\left(\begin{array}{cccc}
1 & T & 0 & 0 \\
0 & 1 & 0 & 0 \\
0 & 0 & 1 & T \\
0 & 0 & 0 & 1
\end{array}\right),
$$

and $T$ is the sampling interval.

The state vector $\mathbf{x}(k)$ contains the $\mathrm{x}$ - and $\mathrm{y}$ - target positions and velocities, i.e.

$$
\mathbf{x}(k)=\left(\begin{array}{c}
x(k) \\
v_{x}(k) \\
y(k) \\
v_{y}(k)
\end{array}\right) .
$$

The measurements are the $\mathbf{x}$ - and $y$ - target positions, i.e. the measurement matrix $\mathbf{H}$ is given by

$$
\mathbf{H}=\left(\begin{array}{llll}
1 & 0 & 0 & 0 \\
0 & 0 & 1 & 0
\end{array}\right) .
$$

Measurements are affected by noise which is modeled as Gaussian, zero mean, with a certain standard deviation. The noise sequence $\mathbf{w}(k)$ has a covariance matrix

$\mathbf{R}=\operatorname{Cov}(\mathbf{w}(k))=\left(\begin{array}{ll}\sigma_{x}^{2} & 0 \\ 0 & \sigma_{y}^{2}\end{array}\right)$.

The values of the noise uncertainties are taken as $\sigma_{x}=\sigma_{y}=55 \mathrm{~m}$ for all targets. The performance is evaluated based on 50-run Monte Carlo simulations. Fig.2 shows the tracks estimates obtained using the NNF, as well as the actual tracks. In this case, the closet measurement to the center of each validation gate is chosen as the correct measurement. It is clear from Figures 2 that the tracks estimates are far from the actual tracks, thus the NNF is not suitable in this case. Fig. 3 shows the tracks estimates obtained using the proposed fuzzy Kalman filter. It is clear from Fig. 3 that the proposed approach improves the tracks estimates and achieves much better performance than the NNF.

The second example considers the case of a 3 maneuvering targets. The dominant acceleration in deterministic target maneuvers is coordinated turn because targets prefer to 
maintain a high speed when in danger, turning rather than slowing down to avoid danger [5]. The initial state estimates and the corresponding initial covariance matrix are obtained by two points differencing of the measurements with a corresponding covariance matrix as in $[2,8]$. Each target motion is initially in a straight line with constant velocity. The measurements are taken every 0.1 second. After generating 250 measurements ( 25 seconds), the targets institute a $10 \mathrm{~g}$ right turn ( $\mathrm{g}=9.8 \mathrm{~m}^{2} / \mathrm{sec}$ ) and hold the turn for 100 measurements and then return to straight lines motion for an additional 250 measurements (producing 600 measurements in all). The values of the noise uncertainties for the three targets are taken as $\sigma_{x 1}=\sigma_{y 1}=30 \mathrm{~m}, \sigma_{x 2}=\sigma_{y 2}=35 \mathrm{~m}$, and $\sigma_{x 3}=\sigma_{y 3}=40 \mathrm{~m}$. The performance is evaluated based on 50-run Monte Carlo simulations. Figure 4 shows the tracks estimates obtained using the proposed fuzzy Kalman filter as well as the actual tracks for all targets. Figure 4 shows that the proposed approach is efficient in case of maneuvering targets. It is worth noting that the proposed approach determines the fuzzy weights for all targets and measurements based on a single partition matrix which highly reduces the computational complexity compared to conventional fuzzy logic approaches and joint probabilistic data association techniques

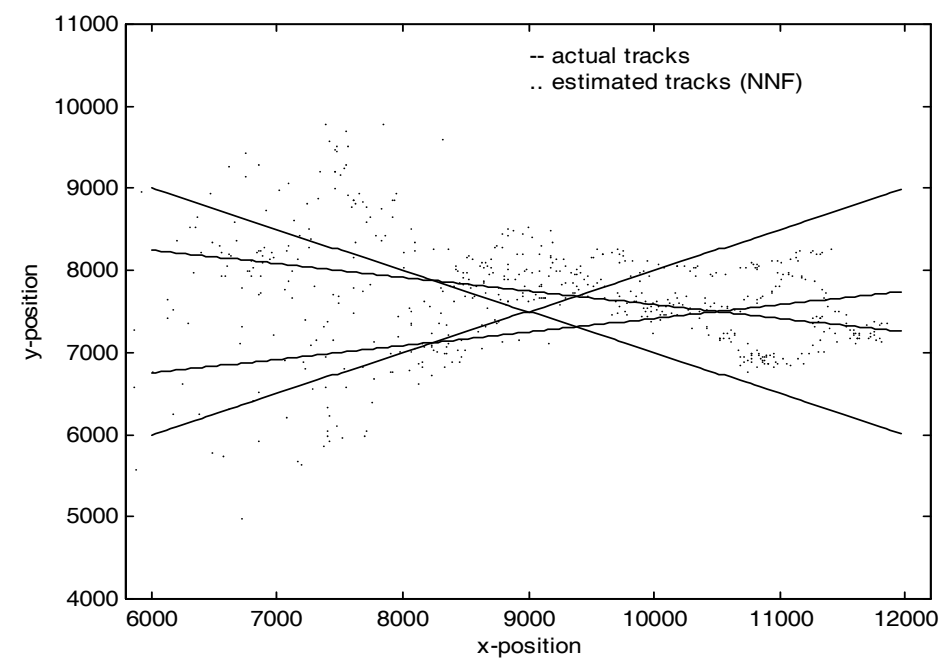

Fig. 2 Performance of a 4-crossing targets tracker using NNF

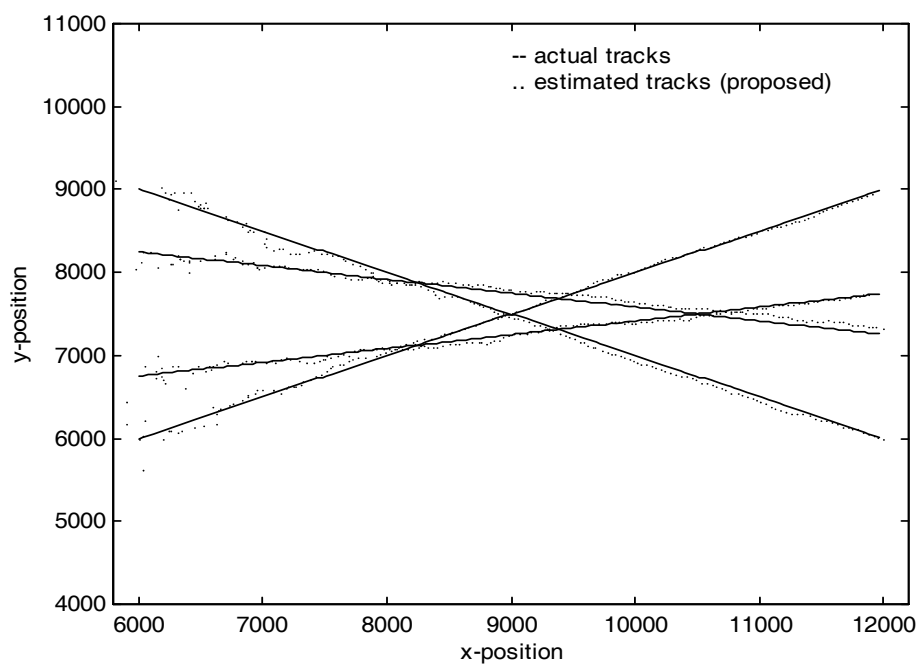

Fig. 3 Performance of a 4-crossing targets tracker using Fuzzy Kalman filter 


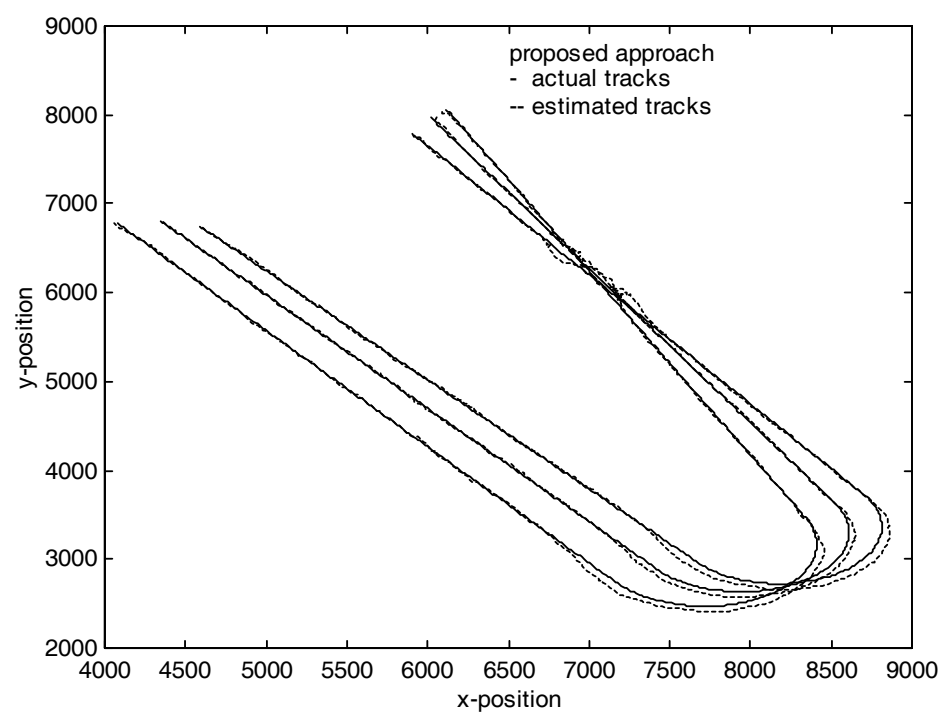

Fig. 4 Performance of a 3-maneuvering targets tracker using Fuzzy Kalman filter

\section{Conclusion}

A new weighting method for tracking multiple targets has been developed. In the proposed method, the probability weights are replaced by fuzzy weights. It obtains the similarity measures between all measurements and all targets and then it updates the target state estimate and the covariance matrix using the fuzzy weights. Each weight represents the similarity measure between a measurement and a target. Unlike many all-neighbor tracking techniques, the proposed approach does not depend on probability measures. Unlike many conventional fuzz tracking techniques, the proposed approach does not use Fuzzy rules. Thus it highly reduces the computational complexity compared to other techniques reported in the literatures. It has been found that the proposed approach improves the tracks estimates and achieves much better performance than the nearest-neighbour filter. The proposed approach can easily be extended to the case of tracking in a cluttered environment. Monte Computer simulations have demonstrated the feasibility and effectiveness of the proposed approach in tracking some typical non-maneuvering and maneuvering targets.

\section{References}

[1] S. Stubberud and K. Kramer, "Data Association for Multiple Sensor Types Using Fuzzy Logic,” IEEE Transactions on Instrumentation and Measurement, vol. 55, No. 6, Dec. 2006, pp. 2292-2302.

[2] Y. Bar-Shalom and T. E. Fortman, Tracking and Data Association, New York, Academic Press, 1988.

[3] Y. Bar-Shalom, Multitarget Multisensor Tracking: Applications and Advances, vol. 2, Norwood, MA: Artech House, 1992.

[4] S. S. Blackman, Multiple-Target Tracking with Radar Applications, Norwood, MA: Artech House, 1986.

[5] Ashraf M. Aziz, "Fuzzy Track-to-Track Association and Track Fusion Approach in Distributed Multisensor-Multitarget Multiple-Attribute Environment,” Signal Processing, vol. 87, No. 6, June 2007, pp. 1474-1492. 
[6] R. Mahler, "PHD Filter of Higher Order in Target Number,” IEEE Transactions on Aerospace and Electronic Systems, vol. 43, No. 4, Oct. 2007, pp. 1523-1543.

[7] J. C. Bezdek, Pattern Recognition with Fuzzy Objective Function Algorithms, Plenum Press, New York, 1981.

[8] I. Turkmen, "IMM Fuzzy Probabilistic Data association Algorithm for Tracking Maneuvering Target,” Expert Systems with Applications, vol. 34, No. 2, Feb. 2008, pp. 1243-1249.

[9] R. A. Singer and A. Kanyuck, "Correlation of Multi-Site Track Data," IEEE Transactions on Aerospace and Electronic Systems, AES-6, March 1970, pp. 180-187.

[10] R. A. Singer and A. J. Kanyuck, “Computer Control of Multiple-Site Track Correlation," Automatica, Vol. 7, 1971, pp. 455-463.

[11] R. A. Singer and K. Behnke, "'Real-Time Tracking Filter Evaluation and Selection for Tactical Applications," IEEE Transactions on Aerospace and Electronic Systems, AES7, No.1, Jan. 1971, pp. 100-110.

[12] D. Willner, B. Chang, and K. P. Dunn, 'Kalman Filter Algorithms for a Multi-Sensor System," Proceedings of IEEE Conference on Decision and Control, Dec. 1976, pp. 570-574.

[13] A. M. Haimovich, J. Yosko, R. Greenberg, M. Parisi, and D. Becker, 'Fusion of Sensors With Dissimilar Measurement/Tracking Accuracies," IEEE Transactions on Aerospace and Electronic Systems, Vol. 29, No.1, Jan. 1993, pp. 245-250.

[14] F. R. Castella, ' Theoretical Performance of a Multisensor Track-to-Track Correlation Techniques," IEE Proceedings on Radar, Sonar and Navigation, Vol. 142, No. 6, Dec. 1995, pp. 281-285.

[15] Y. Bar-Shalom and X. Rong Li, “Multitarget Multisensor Tracking: Principles and Techniques," IEEE AES Systems Magazine, Feb. 1996, pp. 41-44.

[16] Y. Bar-Shalom and X. Rong Li, "Multitarget Multisensor Tracking: Principles and Techniques," IEEE Control Systems Magazine , Feb. 1996, pp. 93-96.

[17] T. Wigren, et al., "Operational Multisensor Tracking for Air Defense," First Australian Data Fusion Symposium, ADFS'96, pp. 13-18.

[18] Jaco Vermaak, Simon J. Godsill, and Patrick Perez, “'Monte Carlo Filtering for MultiTarget Tracking and Data Association," IEEE Transactions on Aerospace and Electronic Systems, Vol. 41, No. 1, Jan. 2005, pp.309-332. 